\title{
LA ARGUMENTACIÓN EN EL DEBATE: UNA ILUSTRACIÓN SOBRE LA APLICACIÓN DE LA RETÓRICA PARA EL ESTUDIO DEL RAZONAMIENTO EN EDUCACIÓN DE ADULTOS
}

\author{
Beatriz Macías Gómez-Estern, \\ José A. Sánchez Medina ${ }^{6}$
}

\begin{abstract}
RESUMEN
El objetivo de este trabajo es describir e ilustrar el empleo de distintos tipos de argumentos en un escenario comunicativo como es el del grupo de discusión en el marco de la educación de adultos. Mostraremos la pertinencia del estudio de los modos de deliberación en relación con los modos de argumentación en el debate y pasaremos a continuación a una descripción de la arquitectura de los argumentos, desplegados por un grupo de mujeres asistentes a un curso de alfabetización de adultos, extraídos de una investigación llevada a cabo en Sevilla, España. Encontramos que el nivel educativo influye en el modo en que estas mujeres argumentan en torno a un tema de su interés. En nuestro trabajo adoptamos una perspectiva retórica en el estudio de los procesos cognitivos.
\end{abstract}

Palabras clave: razonamiento, argumentación, retórica, educación de adultos, modos de pensamiento.

\begin{abstract}
The aim of this work is to describe and illustrate the use of different kinds of arguments in a communicative setting as the focus group. We will show the pertinence of studying ways of deliberation in relation to ways of arguing in the debate. After that, we will analyse the architecture of arguments displayed by a group of women attending an adult literacy course, extracted from a research ran in Seville (Spain), about the relation about adult literacy and different ways of reasoning. We found that the educative level influences the way these women argue about a topic they are interested on. We will then use a rhetoric perspective in the study of cognitive processes.
\end{abstract}

Key words: reasoning, argumentation, rhetoric, adult literacy, ways of thinking.

Recibido: 13 de marzo de 2009 Aceptado: 23 de octubre de 2009

6 Departamento de Ciencias Sociales, Universidad Pablo de Olavide, Sevilla. Carretera de Utrera km 1, Sevilla 41013 Sevilla, España. Email: bmacgom@upo.es. Teléfono: 954977 505. Fax: 954349199 


\section{INTRODUCCIÓN}

En este trabajo estudiaremos los procesos comunicativos que se producen en un contexto determinado como es el del grupo de discusión en el contexto de la Educación de Adultos. En la consecución de este objetivo empezaremos por delimitar a qué nos referimos con nuestra apuesta por una dimensión retórica en el estudio del pensamiento. Esta opción metodológica es fruto de una serie de decisiones epistemológicas acerca de la naturaleza del razonamiento que, como otras funciones mentales, es considerado desde la Psicología Tradicional como un proceso psicológico interno ¿Por qué entonces hacemos uso de una situación social, como es el grupo de discusión, para analizar los procesos de razonamiento? Responder a esta pregunta será el objetivo de la primera parte de nuestro trabajo. A continuación pasaremos a ilustrar las ideas expuestas anteriormente, analizando los enunciados desplegados por un grupo de mujeres participantes en un grupo de educación de adultos de Andalucía, España. Estudiaremos pues la arquitectura de los argumentos empleados en el grupo de discusión con relación a los modos de razonamiento implicados.

\section{La tradición del razonamiento formal}

En Psicología existe actualmente un debate abierto con relación a cuál es la estructura y el contenido del razonamiento humano. Numerosas tradiciones psicológicas abordan este problema desde marcos teóricos bien diferenciados. Por un lado, están las que conciben el razonamiento como un proceso eminentemente lógico-formal $\mathrm{y}$, por otro, están las que lo entienden como un proceso de razonamiento informal basado en otros principios diferenciados de la lógica formal (Carretero y Asensio, 2004). A continuación expondremos brevemente cada una de estas perspectivas.

Tradicionalmente, la Psicología Cognitiva del Procesamiento de la Información ha concebido el razonamiento como un proceso que consiste en la aplicación de reglas eminentemente lógicas, y, por tanto, la lógica es presentada como la mejor manera de deliberar y de resolver conflictos. Esto es debido a que se ha identificado el razonamiento con resolución de problemas, de manera que ha existido un gran interés por descubrir las reglas que supuestamente el sujeto debe seguir para que su proceso de deliberación sea exitoso. Dentro de la Psicología Cognitiva es posible distinguir diferentes planteamientos en la forma de entender la naturaleza de las reglas que emplean los sujetos en la resolución de problemas. A continuación expondremos brevemente los más sobresalientes.

El primero de estos planteamientos está formado por un grupo de investigadores del Procesamiento de la Información que defienden que el razonamiento se articula a través de la denominada lógica natural. Esta lógica está constituida por un conjunto de reglas abstractas y formales que son utilizadas para inferir una conclusión a partir de unas premisas determinadas. Para estos autores las reglas a través de las cuales se realizan estas inferencias están basadas en la lógica formal y operan con independencia del contenido del problema y del contexto donde se desarrollan, por lo que actúan a nivel puramente sintáctico (Rips, 1989; Braine y O’Brien, 1991). 
Las primeras respuestas a los modelos más formales del razonamiento aparecieron cuando, ante problemas reales planteados en el laboratorio, los sujetos cometían errores en el razonamiento lógico debido fundamentalmente a que el contenido de la tarea parecía afectar a su resolución. Para los autores que consideran la aplicación de reglas formales como el substrato subyacente en el razonamiento humano, los errores son los razonamientos que no se ajustan a los corsés de dicha lógica proposicional. En los experimentos citados los errores eran la norma y no la excepción. Se produjeron fundamentalmente dos reacciones ante estos datos. Por un lado, los autores más radicales dentro de la corriente del razonamiento formal desecharon el estudio de estos errores y continuaron rastreando las reglas formales en el razonamiento humano, aunque teniendo en cuenta la importancia del contenido en la resolución de la tarea. En este sentido destacan los Modelos Mentales de Johnson-Laird (1990). Este autor mantiene que el razonamiento de los individuos se caracteriza por la lógica-proposicional. Con ello no se descarta que existan otras formas de razonamiento empleadas sobre todo en la vida cotidiana. Sin embargo, estos procesos no constituyen su objeto de estudio por plantear obstáculos al estudio de los procesos lógicos que son los que definen el razonamiento genuinamente humano. Según este autor, lo que realmente caracteriza el razonamiento de los individuos es su estructura lógica (Rips, 1989; Evans, 1991).

Una segunda reacción ante los datos obtenidos en situaciones de laboratorio donde los sujetos no daban una solución lógica a los problemas planteados, es un conjunto de estudios que inciden en que el razonamiento sigue unas reglas específicas sensibles al contenido. En esta línea se engloba a aquellas perspectivas que proponen la posibilidad de que las reglas del razonamiento sean específicas según el contexto en el que se aplican (Morris y Nisbett, 1993); o que en vez de reglas, lo que usamos son heurísticos o reglas por omisión (Kahneman y Tversky, 1982; Tversky y Kahneman ,1986).

El problema que se plantea entonces es que, si en ocasiones no solemos razonar de forma lógica en situaciones experimentales resolviendo problemas de tipo lógico, difícilmente lo haremos en situaciones de comunicación cotidiana. En este sentido, la lógica no parece que deba ser considerada como el primer y mejor mecanismo para la resolución de problemas cotidianos y, sobre todo, de carácter socio-moral. En los últimos tiempos han surgido intentos por explicar el razonamiento en situaciones de la vida cotidiana cuya organización está alejada de las estructuras lógicas bajo el término de razonamiento informal (Carretero, Almaraz y Fernández, 1995).

\section{El razonamiento informal}

Uno de los principales problemas con que nos encontramos al acercarnos al concepto de razonamiento informal es la heterogeneidad de posturas existentes en cuanto a su concepción. Desde algunas de estas posturas se entiende que las personas, ante una situación cotidiana, buscan las premisas que subyacen al problema planteado. Esto es, los sujetos tratan de desentrañar las relaciones implícitas entre los elementos del problema para, a partir de ese momento, aplicar un tipo de razonamiento que se asemeja mucho al mencionado razonamiento formal. Realmente el tipo de razonamiento seguiría siendo el mismo que el 
planteado desde las posiciones que estudian el razonamiento formal, pero aplicado en este caso a situaciones cotidianas.

Es a esta corriente dentro del razonamiento informal a la que Antaki (1994) se refiere cuando afirma que, pese a su desligamiento de la idea de las reglas formales como el único instrumento del pensamiento racional humano, los teóricos del razonamiento informal se encuentran todavía bastante comprometidos con algunas de sus asunciones. Según Antaki (1994), las investigaciones realizadas en el marco del razonamiento informal guardan cierto "parecido de familia" con las del razonamiento formal. Esta similitud deriva de que ambas corrientes consideran que la relación entre unas premisas y una conclusión es la base del razonamiento. Si las premisas son "verdaderas" la conclusión debe serlo también, siendo de nuevo el contenido de las premisas irrelevante para la validez del razonamiento.

¿Cuál es entonces la diferencia entre las posturas que defienden un razonamiento lógico y las que flexibilizan esta idea proponiendo que el razonamiento humano es informal desde esta perspectiva? Algunos teóricos del razonamiento informal, aunque comparten que la estructura del razonamiento de la lógica formal es clara e inequívoca, reconocen que ésta es difícil de aplicar a los problemas que el sujeto afronta en la vida cotidiana por dos razones. La primera, los términos que denotan las relaciones en la lógica formal y en el lenguaje cotidiano (como "si", "no", "entonces", “implica", etc.) adoptan significados distintos en cada uno de estos contextos. La segunda, la validez de un argumento lógico no podrá nunca ser alterada una vez alcanzada la conclusión de unas premisas verdaderas. Éste no es el caso del razonamiento usado en contextos cotidianos, en el que se dan procesos inductivos, por ejemplo, susceptibles de "falsación" por el descubrimiento de alguna información no incluida en la primera versión.

La vertiente del razonamiento informal que nos interesa ha tenido su tradicional campo de desarrollo en el contexto de la educación en la escuela del pensamiento crítico. Esta tradición se ocupa de la enseñanza de la aplicación de la lógica formal a problemas más o menos académicos. Sin embargo, esta tarea ha resultado más ardua de lo que los teóricos de la lógica pudieran esperar. Morton (1988) observó que, después de mucho entrenar a estudiantes universitarios inteligentes en las técnicas de la lógica informal éstos no alcanzaban a usarlas con fluidez. También observó que al buscar ejemplos para usar en sus clases de lógica informal tenía que rechazar la mayoría de los argumentos que encontraba en periódicos y revisas, a los que consideraba, por otra parte, una prosa argumentativa perfectamente clara y directa (Fisher,1988).

Hasta ahora nos hemos centrado en una de las perspectivas más restrictivas de entre las que estudian el razonamiento informal, pero como hemos indicado anteriormente éste es un concepto que engloba posturas muy heterogéneas. Fernández y Carretero (1995) señalan:

"La capacidad de argumentar, de proporcionar razones que apoyen una afirmación o una conclusión, de valorar posiciones contrarias, de elaborar contrargumentos y/o posiciones 
alternativas, parecen formar parte, aunque, probablemente, no de una manera exclusiva, del razonamiento informal." (p. 41).

La definición de Fernández y Carretero (1995) del razonamiento informal remite directamente a una concepción comunicativa y retórica de los argumentos. Desde este punto de vista, la forma de estudiar cómo las personas razonan es mediante el análisis de los procesos comunicativos y las interacciones sociales. En estos procesos es preciso construir argumentos que persuadan a una audiencia, defender una determinada posición y justificarla frente a las críticas.

Pero, ¿a qué nos referimos cuando hablamos de argumentación? En la definición de argumentación existen diferencias referidas a cuáles deben ser los criterios de validación de los argumentos, es decir, cuáles son los criterios por los que un argumento es considerado como aceptable o convincente. Elegimos la propuesta de Toulmin , Riecke y Janick, (1979) por considerar el contexto como determinante en su validación.

Para Toulmin el proceso argumentativo, cuyas características principales tienen que ver con la justificación y la crítica, se produce en contextos sociales. La crítica se da cuando se considera que alguna norma social ha sido violada, y la justificación, en situaciones que provocan críticas o que pudieran ser potencialmente evaluadas negativamente. La crítica y la justificación son pues procesos socialmente situados, dependientes de los marcos de valores de las distintas comunidades. Esta postura plantea un argumento no dependiente exclusivamente del individuo que lo esgrime, sino co-construido en gran parte por las normas sociales y por el contexto concreto de interacción en el que se esgrime. Esta implicación es importante ya que constituye un giro radical en la concepción de los fenómenos argumentativos en general, desde una postura tradicional que los considera como productos de la deliberación individual a una postura que plantea una fuerte influencia de lo social en su construcción.

\section{La retórica como instrumento para el estudio de la argumentación y el razonamiento}

El estudio de los argumentos como vía para el análisis de los procesos de deliberación y razonamiento no es algo nuevo en las ciencias sociales. Autores como Vygotski (1991), Mead (1974) o Bakhtin (1981) han enfatizado de distinto modo la doble orientación del discurso, mostrando que éste no sólo se dirige a un auditorio externo, sino que afecta también al mismo hablante en su plano interno. Más recientemente, desde el constructivismo social, también se ha apoyado esta idea. Billig (1987) citando a Isócrates sostiene que "los mismos argumentos que usamos para persuadir a los demás cuando hablamos en público, los usamos también cuando deliberamos en nuestros pensamientos" (p.111). Asume que aprender a argumentar constituye una parte importante del aprender a pensar. Perelman y Olbrechts-Tyteca (1994) definen la deliberación íntima como un tipo particular de argumentación, en el que las opiniones puestas en juego son generadas por una misma persona. El acuerdo con uno mismo, la convicción de algo, no es para ellos más que un ejemplo concreto de la categoría más amplia que es el acuerdo con los demás. Por eso afirman que el análisis de la argumentación dirigida a los demás nos llevará a entender mejor la deliberación íntima. 
Debemos hacer especial mención a la retórica, disciplina que durante siglos se ha ocupado del estudio de los distintos tipos de argumentos empleados para persuadir a una audiencia determinada. Proponemos hacer uso de esta tradición del conocimiento y los desarrollos alcanzados en ella para el análisis psicológico de los argumentos y la deliberación en variados tipos de contextos (para un recorrido histórico sobre las distintas acepciones que ha adoptado el concepto de retórica véase Foss, Foss y Trapp, 1991; Perelman y Olbrechts-Tyteca, 1994 y Frogel, 2004).

En el ámbito de la psicología más reciente, numerosos autores pertenecientes a tradiciones psicológicas muy diversas han relacionado pensamiento y deliberación con formas de argumentación (Billig, 1987, desde el constructivismo social; Wertsch ,1998 y Ramírez,1995 desde la Psicología Histórico Cultural). En toda argumentación se ve implicado el proceso de pensamiento en cuanto que al sujeto le corresponde determinar cuáles deben ser los elementos y el modo de organizarlos para que un argumento resulte convincente. Por otro lado, el interlocutor del acto argumentativo debe deliberar sobre la veracidad o confiabilidad de los argumentos que le son presentados.

A diferencia de la lógica, que pretende convencer de una evidencia, de una verdad, que una vez asumida es irrefutable; la retórica opera en el campo de la opinión, en el que la función del hablante es persuadir al auditorio con el propósito práctico de que adopte la perspectiva propia. La lógica está dirigida a un auditorio universal que necesariamente sucumbe ante la evidencia. Cualquier ser racional debe admitir la verdad de una demostración lógica referida a hechos contrastados e inamovibles. La necesidad de una conclusión derivada del razonamiento lógico hace que sea innecesaria la argumentación, ya que lo "dado" y verdadero no plantea cuestionamiento ni refutación. La retórica, sin embargo, obtiene adhesiones con intensidad variable en el auditorio y se nutre de proposiciones probables y verosímiles, que han de ser aceptadas razonablemente (Van Eemeren, 2001).

Estas disparidades en los objetivos de la retórica y la lógica dejan entrever la importancia diferencial que el auditorio adquiere para cada una de ellas. Una de las aportaciones más celebradas a la nueva retórica planteada por Perelman y Olbrechts-Tyteca (1994) tiene que ver con el papel de cuasi co-autoría que se le confiere a la audiencia en la construcción del discurso retórico. Estos autores ponen un énfasis especial en la idea de que todo argumento va dirigido a un auditorio, esté presente físicamente o no. Esto hace al discurso vulnerable a las críticas potenciales del auditorio, críticas que el orador debe prever y tener en cuenta en la dialogicidad de su argumento. Será también este auditorio el que valore la credibilidad del argumento presentado, por lo que los criterios de validez de la retórica dependen en gran medida de los valores, marcos y disposición del auditorio al que el discurso va dirigido.

\section{Arquitectura de los argumentos: razonamiento teórico versus práctico}

Una vez justificado el uso de la retórica para el estudio del razonamiento, pasaremos a explorar el armazón de los argumentos con la intención de descubrir los distintos tipos de 
argumentos que pueden ser empleados en un grupo de discusión partiendo de una visión amplia de lo que significa argumentar.

Tradicionalmente en retórica se han distinguido dos tipos fundamentales de argumentos: unos basados en generalizaciones y otros en particularidades. Jonsen y Toulmin (1988) diferencian entre los argumentos analíticos o teóricos, basados en premisas generales a partir de las cuales se obtiene una conclusión; y los argumentos sustanciales o prácticos, en los que a partir de un caso particular se infiere una conclusión no contenida en las premisas. A algo similar se refieren Perelman y Olbrechts-Tyteca (1994) cuando diferencian entre argumentos que denominan cuasi lógicos y argumentos basados en la estructura de lo real.

Según Perelman y Olbrechts-Tyteca (1994) el razonamiento teórico guarda cierta similitud con la lógica, aunque el mecanismo por el que funciona es diferente. El argumento práctico, a diferencia del argumento teórico, no posee apariencia lógica ya que se apoya en un caso particular, a partir del cual se infiere una conclusión. Su fundamento se encuentra en la estructura de la realidad sirviéndose de ésta para establecer una similitud entre juicios admitidos y aquellos que se intenta promover (Millgram, 2001). La persuasión se alcanza mediante el aumento de la presencia en el discurso de una imagen concreta proporcionada por lo realista del caso particular (Walton, 2000).

El razonamiento por analogía es un ejemplo de argumentación basado en lo real (Shelley, 2004). La fórmula más general de la analogía tipo es: A es a $\mathrm{B}$ lo que $\mathrm{C}$ es a $\mathrm{D}$. La analogía es una semejanza de relación más que una relación de semejanza, en la que un tema o conclusión (A es a B), es esclarecido por su semejanza con un foro (C es a D) más conocido por el auditorio que el primero. El modelo de analogía presentado constituye el tipo más prototípico o genérico de ellos. Se pueden dar diferentes versiones de esta forma por omisión de alguno o varios de los elementos incluidos en la relación, como es el caso de la metáfora.

Las analogías y las metáforas son muy usadas en todos los ámbitos de nuestra vida. Pueden ser considerados como instrumentos muy útiles para la exploración de nuevos campos de conocimiento o incluso para la re-interpretación de otros ya explorados. Lakoff y Johnson (1980) afirman que las metáforas juegan un papel central en nuestro sistema conceptual. Aquello que pensamos, lo que hacemos y decimos en nuestra vida cotidiana están en gran medida sustentados por metáforas del tipo una discusión es una guerra, o el tiempo es dinero. La mayoría de ellas están ancladas en una relación del mundo físico, a partir de la cual, por analogía, se explican o interpretan conceptos no físicos más complejos. Un ejemplo clásico del análisis de la influencia de las metáforas en nuestra concepción del mundo es el ofrecido por Reddy (1979) en relación con la comunicación, que es entendida tradicionalmente con base en la "metáfora de la transmisión" ("conduit metaphor").

Con lo dicho hasta el momento de los distintos tipos de argumentos queremos defender la consistencia y persuasividad retórica de ambos tipos descritos: los argumentos teóricos o cuasilógicos y los prácticos. Ambas formas de argumentar son necesarias para una explicación, descripción y aclaración (Antaki, 1994) de circunstancias o estados mentales. El poder 
persuasivo de un tipo u otro de argumento vendrá determinado por la tradición de uso que cada uno tenga en distintos escenarios sociales.

\section{Tipos de argumentos y modos de pensamiento}

Podemos relacionar los tipos de argumentos estudiados desde la retórica con la distinción establecida desde la psicología por Bruner (1988) entre dos modos de pensamiento: el modo proposicional o paradigmático y el narrativo. Estas modalidades son complementarias en el pensamiento humano pero irreductibles la una a la otra. La modalidad paradigmática requiere un uso descontextualizado de los instrumentos semióticos, un descentramiento en las características concretas de la situación y el contexto inmediato, transcendiendo lo particular para entrar en el mundo de los argumentos universales y generalizables a toda una comunidad. En cuanto el pensamiento narrativo, este se caracteriza por ocuparse de: "las intenciones y acciones humanas y de las vicisitudes y consecuencias que marcan su transcurso. Trata de situar sus milagros atemporales en los sucesos de la experiencia y de situar la experiencia en el tiempo y el espacio" (Bruner, 1988, p.24).

Organizamos y creamos muchos aspectos de nuestra existencia en torno a historias, narraciones que construimos a partir de hechos que nos cuentan, oímos, vemos o imaginamos. Normalmente en estas parcelas de nuestra experiencia, no utilizamos un pensamiento paradigmático, como haríamos, por ejemplo, para resolver un problema matemático (Bruner, 1992; Esteban, 2002). Así como la razón está gobernada por una lógica única e ineludible, las historias son muchas y variadas (White, 1981).

Los modos de pensamiento ligados a instrumentos psicológicos están relacionados con los escenarios de actividad en los que estos últimos son usados, por lo que los modos de pensamiento que generan, lo están también. Las actividades pueden ser comunes en distintas culturas, así como una persona o grupo es partícipe diariamente en gran número y tipos de actividades como por ejemplo la actividad escolar y la cotidiana (Tulviste, 1992; Luria 1987; Scribner y Cole, 1081; Olson y Torrance, 1991).

\section{Aprender a debatir, aprender a pensar: una ilustración}

El debate es considerado tradicionalmente como un evento comunicativo altamente sofisticado que requiere del dominio (mastering) de las funciones retóricas del lenguaje. Sin embargo, esta idea puede cuestionarse. El debate de ideas, conceptos y puntos de vista ha sido presentado y defendido en numerosas ocasiones como un excelente instrumento didáctico para promover la adquisición de conocimiento y como vía de desarrollo del pensamiento (Cros, 2002). Ejemplos de este tipo pueden constituir los trabajos de Palincsar y Brown (1988) sobre el método de la enseñanza recíproca o las propuestas de Freire (Freire y Macedo, 1989) sobre el método de la palabra generadora. Los usuarios de estos programas, se involucran en prácticas dialógicas que implican el debate, argumentación y defensa de los propios puntos de vista, incluso antes de que dominen la tecnología de la lecto escritura (Davies, 2003; Relaño y Macías, 2002; Rosemberg C.R. y Borzone A.M. ,2001). 
Para ilustrar estos dos modos de argumentar empleados en el debate mostraremos una serie de ejemplos. En una investigación desarrollada por el Laboratorio de Actividad Humana estudiamos los modos de argumentar de mujeres que asistían a centros de educación de adultos (Cala, 2002). En este caso nos preguntábamos si el uso de uno u otro modo de argumentar, es decir, si el uso de una modalidad u otra de pensamiento, estaba asociado al grado de escolarización de los individuos. Para responder a esta pregunta propusimos a grupos de mujeres que debatiesen acerca del papel que la mujer jugaba en la sociedad y si hombres y mujeres podían desarrollar por igual las mismas tareas, laborales y domésticas. De estos debates están extraídas las siguientes intervenciones:

1. "...Yo tengo una amiga, que está su marido en correos..., no es amiga mía, es amiga de mi hija, pero eso es igual, y cuando viene su marido es cuando se ponen a bañar al niño, y cuando... hasta que no viene él no bañan al niño, ni hace esto ni hace lo otro. Todo, todo lo comparten..."

2. "... eso, pero yo no lo veo, partiendo de lo que decía ella del trabajo entre los dos, aquí lo que pasa es que no estamos acostumbrados a eso, pero si desde el principio estuviésemos acostumbrados, el salir la mujer a la calle era tan normal como para el hombre, porque al llegar a la casa las faenas serían igual de uno que de otro..."

Los enunciados 1 y 2 pueden tomarse como ejemplos típicos de los dos modos de argumentar que hemos estado presentando, el argumento práctico y el argumento teórico. En el primero de ellos la hablante argumenta contando una historia, usando un argumento práctico. Narra unos hechos que emplea para convencer a su audiencia de que es posible que hombres y mujeres compartan, por igual, las tareas del hogar. Si nos fijamos con detenimiento podemos encontrar fragmentos del enunciado que en principio nos podrían parecer ilógicos o al menos innecesarios. Por ejemplo, cuando la mujer comenta: "Yo tengo una amiga, que está su marido en correos..., no es amiga mía, es amiga de mi hija...”. Esta afirmación parece innecesaria respecto de la posición que la hablante va a defender. Es decir, en principio es indiferente que los hechos que narra se refieran a una amiga suya o a una amiga de su hija. Si reflexionamos desde este punto de vista probablemente nos estemos dejando llevar por un modo de pensamiento que se ajusta al segundo enunciado. Expliquemos esto. Al presuponer que es innecesario este fragmento del argumento asumimos que dos experiencias particulares (la de madre e hija en este caso) son intercambiables, en cuanto que no se consideran como experiencias particulares sino como ejemplos que pueden ilustrar otras muchas experiencias. Las estamos tratando como categorías. Sin embargo, para la mujer que argumenta este hecho no es así. La capacidad retórica, la fuerza argumentativa de su enunciado radica en la verosimilitud del relato que sirve de argumento. Desde esta perspectiva, si uso una historia para argumentar, su fuerza persuasora será mayor cuanto más real sea la historia. Los datos que desde una perspectiva pueden resultar accesorios, desde la otra juegan un papel crítico en el proceso de argumentación. No cabe duda de que un relato dispondría de menor fuerza persuasiva si se hubiese articulado, por ejemplo, del siguiente modo: "Supongamos una mujer que trabaja y que su marido....". 
El enunciado 2 es en cambio una buena argumentación que utiliza modos de pensamiento proposicionales, del razonamiento teórico. Aquí no se cuenta una historia, sino que se establecen unos hechos "lo que pasa es que no estamos acostumbrados a eso..." [el trabajo compartido entre hombres y mujeres] una hipótesis “...si desde el principio estuviésemos acostumbrados, el salir la mujer a la calle era tan normal como para el hombre...” y a partir de la misma unas conclusiones (en el plano de la opinión) "al llegar a la casa las faenas serían igual de uno que de otro”. En este caso la argumentación no presenta la estructura de un relato, sino más bien se ajusta a un proceso de deducción. Pero no sólo cambia la estructura del enunciado, sino también el tipo de referente utilizado. En el primer caso, y como hemos comentado, el establecimiento de la particularidad de los personajes tratados resulta muy importante de cara a conseguir capacidad persuasora. En el segundo, esta capacidad radica en que lo dicho es aplicable, no a una persona concreta, sino a cualquier persona que viva en este entorno cultural (que es el responsable de que no estemos acostumbrados a que hombre y mujer compartan las tareas del hogar). Mientras en el primer caso se usaban expresiones como mi hija, su amiga, mi marido, en el segundo estas son sustituidas por otras como la mujer o el hombre. En el primer caso domina la particularización, en el segundo la generalización.

Hasta ahora hemos ilustrado distintos tipos de argumentos usados en los debates en grupos de alfabetización de adultos. Haremos a continuación una breve reflexión sobre los usuarios de los mismos. Si bien ninguna de las formas de argumentación era de uso exclusivo por ninguno de los grupos de alfabetización, los enunciados del tipo 1 eran utilizados fundamentalmente por las mujeres de los niveles educativos inferiores, mientras que los enunciados del tipo 2 correspondían frecuentemente a intervenciones de las mujeres de mayor experiencia escolar. Las investigaciones que realizamos sobre estos modos de argumentar parecían apuntar a que los modos de argumentación basados en un razonamiento teórico resultaban casi exclusivos de hablantes con amplia experiencia escolar, mientras que los basados en un razonamiento práctico, aunque eran más frecuentes en las mujeres de baja experiencia escolar, también eran usados, con menor frecuencia, por mujeres de elevada experiencia escolar. Estos datos nos conducen a dos reflexiones importantes:

1. el acceso a modos de razonamiento teórico está ligado a la experiencia escolar.

2. el acceso a modos de razonamiento teórico no supone dejar de usar modos de razonamiento práctico.

No cabe duda de que se podría profundizar más en la estructura de la argumentación en el debate. Nosotros nos hemos centrado en los aspectos más generales. Dentro de los modos teóricos y prácticos existen innumerables modos de articular un argumento, pero todos ellos se ajustan a los principios y moldes que aquí hemos presentado. Igualmente podríamos haber citado otros modos de pensamiento y argumentación pero de cara a la simplicidad nos hemos limitado a éstos. Como conclusión, y siguiendo a Toulmin y Cols. (1979) señalaremos que los modos de argumentar y explicar están en estrecha relación con las prácticas culturales de los grupos sociales que usan tales argumentos y explicaciones. 


\section{REFERENCIAS BIBLIOGRÁFICAS}

ANTAKI, C. (1994): Explaining and Arguing. The social organization of accounts. Londres: Sage.

BAKHTIN, M. (1986): Speech genres and Other Late Essays. Austin, Texas: University of Texas Press.

BILLIG, M. (1987): Arguing and thinking. Cambridge, Mass.: Cambridge University Press.

BRAINE, M. D. S. Y O'BRIEN, D. P. (1991): A theory of if: A lexical entry, reasoning program, and pragmatic principles. Psychological Review, 98, 182-203.

BRUNER, J. (1988): Realidad mental, mundos posibles. Barcelona: Gedisa.

BRUNER, J. (1992): Actos de significado. Madrid: Alianza Psicología Minor.

CALA, M. J. (2002): Género, grado de escolarización y actitudes. Modos de argumentar y pensar, Cultura y Educación 14 (3), 327-342.

CARRETERO, M.,ALMARAZ, J. Y FERNÁNDEZ, P. (1995): Razonamiento y comprensión. Valladolid: Simancas Ediciones S.A.

CARRETERO, M. Y ASENSIO, M. (2004): Psicología del Pensamiento. Madrid: Alianza.

CHENG, P. W. y HOLYOAK, K.J. (1985): Pragmatic reasoning schemas. Cognitive Psychology, 17, 319-416.

CROS, A. (2002): Elementos para el análisis del discurso de las clases, Cultura \& Educación, 14 (1), 81-97.

DAVIES, C. E. (2003): How English-learners joke with native speakers: an interactional sociolinguistic perspective on humor as collaborative discourse across cultures, Journal of Pragmatics, 35, 1361-1385.

ESTEBAN, J. (2002): La teoría narrativa de Bruner y sus implicaciones para una pedagogía hermenéutica, Cultura \& Educación, 14 (3), 253-265.

EVANS, J. St. B. T. (1991): Theories of human reasoning: the fragmented state of the art, Theory \& Psychology, 1, 83-105.

FERNÁNDEZ, P y CARRETERO, M (1995): Perspectivas actuales en el estudio del razonamiento. En CARRETERO, M., ALMARAZ, J. Y FERNÁNDEZ, P. (Eds). Razonamiento y comprensión. Valladolid: Simancas Ediciones S.A. 
FISHER, A. (1988): Critical thinking. Proceedings of the First British Conference of Informal Logic and Critical Thinking. Norwich: University of East Anglia Press.

FOSS, S.K. FOSS, K.A. y TRAPP R. (1991): Contemporary perspectives on rhetoric. Prospect Heights, Ill.: Waveland Press.

FREIRE, P. y MACEDO, D. (1989): Alfabetización. Lectura de la palabra y lectura de la realidad. Barcelos: Paidós-MEC.

FROGEL, S. (2004): Philosophical argumentation: logic and rhetoric, Argumentation, 18, 171-188.

JOHNSON-LAIRD, P.M. (1990): El ordenador y la mente: introducción a la ciencia cognitiva. Barcelona: Paidós.

JONSEN, A. Y TOULMIN, S. (1988). The Abuse of Casuistry: A History of Moral Reasoning. Berkeley: University of California Press.

KAHNEMAN, D. Y TVERSKY, A. (1982): The simulation heuristic. En D. KAHNEMAN, P. SLOVIC Y A. TVERSKY. (Eds.), Judgment under uncertainty: Heuristics and biases. Cambirdge, MA: Cambridge University Press.

LURIA, A.R. (1987) Desarrollo histórico de los procesos cognitivos. Madrid: Akal.

MEAD, G.H. (1974): Mind, Self \& Society. From the Standpoint of a Social Behaviorist. Chicago: The University of Chicago Press (version original 1934).

MILLGRAM, E. (ED.) (2001): Varieties of Practical Reasoning. Cambridge Mass.: MIT Press.

MITCHELL, W.J.T. (Ed.): On Narrative. Chicago: The University of Chicago Press.

MORTON, A. (1988): Making arguments explicit: the theoretical interest of practical difficulties. En FISHER, A. (Ed), Critical thinking. Proceedings of the First British Conference of Informal Logic and Critical Thinking. Norwich: University of East Anglia Press.

MORRIS, M. W. Y NISBETT, R. E. (1993). Tools of the trade: Deductive schemas taught in psychology and philosophy. En R. E. Nisbett, (Ed.), Rules for Reasoning. Hove: LEA.

OAKHILL, J.V. y JOHNSON LAIRD, P. N. (1985): Rationality, memory and the search for counterexamples. Cognition, 20, pp. 79-84. 
OLSON, D. y TORRANCE, N. (1991): Literacy and orality. Cambridge, Mass: Cambridge University Press.

PALINCSAR, A. S. Y BROWN A.L. (1988): Teaching and practising thinking skills to promote comprehension in the context of group problem solving. RASE, 9(1), pp. 53-59.

PERELMAN, C. Y OLBRECHTS-TYTECA, L. (1994): Tratado de argumentación. La nueva retórica. Madrid: Gredos.

RAMIREZ, J. D. (1995): Uso de la palabra y sus tecnologías. Buenos Aires: Miño y Dávila Editores.

RELAÑO, M. Y MACÍAS, B. (2002): Argumentation and self-representation in everyday narratives: the logo activity. En VAN EMEREN, H.; BLAIR, A.; WILLARD, C.H. AND HENKEMANS, F.S. (2002): Proceedings of the fifth conference of the International Society for the Study of Argumentation, 115-119.

RIPS, L. J. (1989): The psychology of knights and knaves, Cognition, 31, 85-116.

ROSEMBERG C. R.; BORZONE A. M. (2001): La enseñanza a través del discurso. Estrategias de contextualización y de descontextualización de significados, Cultura \& Educación, 13 (4), 407-424.

SCRIBNER, S. y COLE, M. (1981): The psychology of literacy. Cambridge, Mass: Harvard University Press.

SHELLEY, C. (2004): Analogy counterarguments: a taxonomy for critical thinking, Argumentation, 18, 223-238.

TOULMIN, S.; RIECKE, R.; Y JANICK, A. (1979): An Introduction to Reasoning. Nueva York: Mcmillan.

TULVISTE, P. (1992): On the historical heterogeneity of verbal thought. Journal of Russian and East European Psychology. 30 (1), 77-88.

TVERSKY, A y KAHNEMAN, D (1983): Extensional versus intuitive reasoning: the conjunction falacy in probability judgement. Psychologycal Review, 90 (4), pp. 293-315.

TVERSKY, A. Y KAHNEMAN, D. (1986): Extensional versus intuitive reasoning: the conjunction fallacy in probability judgment, Psychological Review, 90 (4), 293-315.

VAN EEMEREN, F. H. (ED.)(2001): Crucial Concepts in Argumentation Theory. Amsterdam: Amsterdam University Press. 
VOSS, J. F., PERKINS, D.N. Y SEGAL, J. V. (1991): Informal reasoning and education. Hillslade, Nueva Jersey: LEA.

VYGOTSKI, L. S. (1991): La conciencia como problema de la psicología del comportamiento. En L.S. Vygotski: Obras Completas. Vol. I. Madrid: Aprendizaje Visor.

WALLTON, D. (2000): Scare tactics: arguments that appeal to fear and threats. Dordrecht: Kluwer Academic Publishers.

WERTSCH, J. V. (1993): Voces de la mente. Madrid: Aprendizaje Visor.

WERTSCH, J. V. (1998): Mind as action. New York: Oxford University Press.

WHITE, H. (1981): The Value of Narrativity in the Representation of Reality. En MITCHELL, W.J.T. (Ed.): On Narrative. Chicago: The University of Chicago Press. 